Note: This is a draft of a paper submitted for publication. Contents of this paper should not be quoted or referred to without permission of the author(s).

\title{
3-D Measurement of Deformation Microstructure in A1(.02\%) Mg Using Submicron Resolution White X-Ray Microbeams
}

\author{
B. C. Larson, N. Tamura, J.-S. Chung, ${ }^{2}$ G. E. Ice, ${ }^{a}$ J. D. Budai, J. Z. Tischler, W. Yang, \\ H. Weiland, ${ }^{b}$ and W. P. Lowe ${ }^{c}$ \\ Solid State Division, Oak Ridge National Laboratory \\ Oak Ridge, TN 37831
${ }^{a}$ Metals and Ceramic Division, Oak Ridge National Laboratory
${ }^{b}$ Alcoa Technical Center, Alcoa Center, PA
"Howard University, Washington D.C.

December 1999

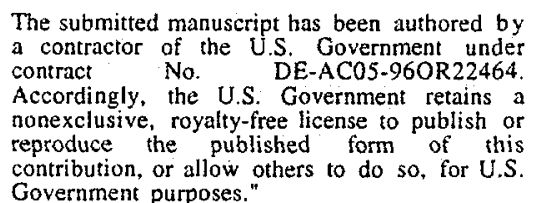

The submitted manuscript has been authored by
a contractor of the U.S. Government under
contract No. DE-AC05-960R22464.
Accordingly, the U.S. Government retains a
nonexclusive, royalty-free license to publish or
reproduce the published form of this
contribution, or allow others to do so, for U.S.
Government puposes." Government purposes."

\author{
prepared by \\ SOLID STATE DIVISION \\ OAK RIDGE NATIONAL LABORATORY \\ Managed by \\ LOCKHEED MARTIN ENERGY RESEARCH CORP. \\ under \\ Contract No. DE-AC05-96OR22464 \\ with the \\ U.S. DEPARTMENT OF ENERGY \\ Oak Ridge, Tennessee
}




\title{
3-D MEASUREMENT OF DEFORMATION MICROSTRUCTURE IN Al(0.2\%)Mg USING SUBMICRON RESOLUTION WHITE X-RAY MICROBEAMS
}

B.C. Larson, N. Tamura, J.-S. Chung, G.E. Ice, J. D. Budai, J. Z. Tischler, and W. Yang, Oak Ridge National Laboratory, Oak Ridge, TN, H. Weiland, Alcoa Technical Center, Alcoa Center, PA, and W.P. Lowe, Howard University, Washington D.C.

\begin{abstract}
We have used submicron-resolution white x-ray microbeams on the MHATT-CAT beamline 7-ID at the Advanced Photon Source to develop techniques for three-dimensional investigation of the deformation microstructure in a $20 \%$ plane strain compressed $\mathrm{Al}(0.2 \%) \mathrm{Mg}$ tri-crystal. Kirkpatrick-Baez mirrors were used to focus white radiation from an undulator to a $0.7 \times 0.7$ $\mu \mathrm{m}^{2}$ beam that was scanned over bi- and tri-crystal regions near the triple-junction of the tricrystal. Depth resolution along the x-ray microbeam of less than 5 microns was achieved by triangulation to the diffraction source point using images taken at a series of CCD distances from the microbeam. Computer indexing of the deformation cell structure in the bi-crystal region provided orientations of individual subgrains to $\sim 0.01$ degrees, making possible detailed measurements of the rotation axes between individual cells.
\end{abstract}

\section{INTRODUCTION}

High-temperature mechanical deformation is an important industrial process for the generation of desired polycrystalline grain structure and forming of structural materials. The disoriented crystalline grains that form the structural units of polycrystalline materials are bounded by grain boundaries and grain boundary intersections. These grain boundaries and triple-junctions severely restrict deformation induced slip processes and, hence, significantly increase the strength of polycrystalline materials relative to that of single crystals. ${ }^{1,2}$ Although highly refined methods have been developed for the control of polycrystalline microstructure, little fundamental understanding exists regarding the detailed mechanisms that lead to the observed microstructures.

$\mathrm{X}$-ray microbeam diffraction represents a powerful new technique for the investigation of orientation and tri-axial strain for individual grains at sub-micron levels. ${ }^{3-5}$ As this technique is developed, it will provide a means for non-destructively studying the local microstructure and stress/strain in bulk materials. In this paper we describe methods for producing submicron $x$-ray beams of white $x$-rays using Kirkpatrick-Baez (K-B) mirrors, and we illustrate their application to 3-D crystallography using a $C C D$ detector. White microbeams are used to obtain 3-D microstructural information through diffraction measurements on a bi-crystal region of an $\mathrm{Al}(0.2 \%) \mathrm{Mg}$ tri-crystal deformed $20 \%$ in plane strain.

\section{EXPERIMENT}

A microbeam diffraction system with interchangeable white and monochromatic radiation has been constructed on the MHATT-CAT beam line at the Advanced Photon Source (APS). ${ }^{3,4}$ ORNL and Howard University have fabricated a (small-offset) monochromator that can be translated in or out of the APS undulator beam to produce co-linear monochromatic or white radiation, respectively. ${ }^{6}$ These (interchangeable) white or monochromatic beams are subsequently focused by crossed K-B mirrors with elliptical figures. As depicted in Fig. 1, 
white/monochromatic undulator radiation is incident on a vertical deflecting mirror with a 130 $\mathrm{mm}$ focal length and a horizontal deflecting mirror with a $60 \mathrm{~mm}$ focal length. Assuming $50 \times 50$ $\mu \mathrm{m}^{2}$ beam defining slits before the mirrors, the converging microbeam has angular spreads of less than a milliradian $(\sim 0.05 / 130$ and $0.05 / 60)$ in the vertical and horizontal directions, respectively. Diffraction patterns are collected on a $1242 \times 1152$ pixel CCD coupled to a phosphor screen by a 1:1 (untapered) fiber optics bundle. The $\sim 22.5$ micron pitch pixels of the CCD correspond to a nominally $28 \times 26 \mathrm{~mm}^{2}$ active area that is typically placed a distance of $\sim$ 30-60 $\mathrm{mm}$ from the sample.

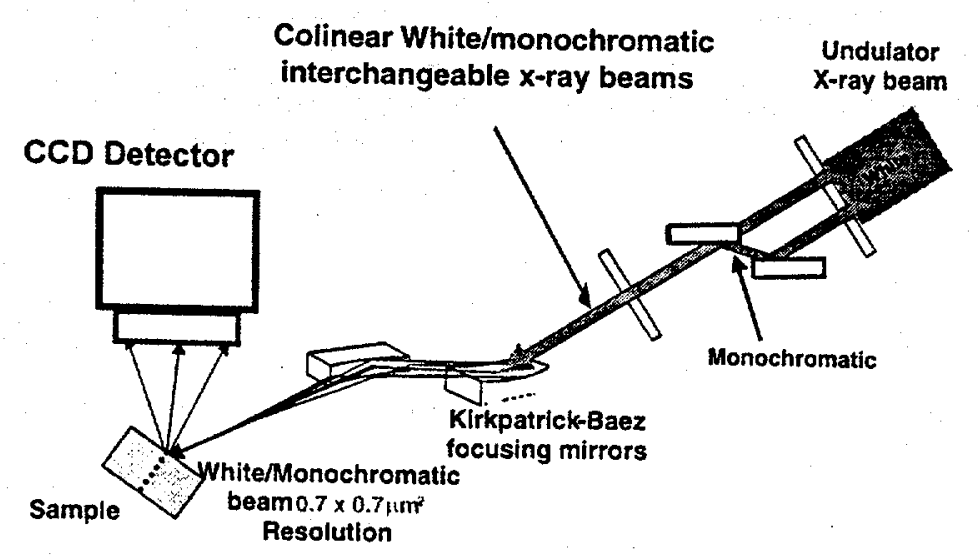

Fig. 1. Schematic overall view of microbeam components. A removable double-crystal monochromator provides white or monochromatic $\mathrm{x}$-rays to crossed K-B mirrors, which focus a submicron beam onto the sample that diffracts into the CCD detector.

Data collection, reduction, and analyses are performed on interactive software that is the subject of ongoing development. Automatic and manual peak searches with two-dimensional Gaussian, Lorentzian, or Pearson VII fitting functions are used to identify and tabulate diffraction features; typically, sub-pixel precision is obtained for diffraction peak positions. Angular and positional calibration of the diffraction system components is accomplished through measurements on a perfect Ge crystal. White beam diffraction measurements for a Ge crystal are used to iteratively refine the parameters by fitting to the undistorted structure and lattice parameter of $\mathrm{Ge}$. In general, the refinement yields angular positions to better than 0.01 degrees and strain resolution of better than a part in $10^{-4}$ for Ge.

The $\mathrm{Al}(0.2 \%) \mathrm{Mg}$ tri-crystal sample used in the measurements presented in this paper was grown at the Alcoa Technical Center and deformed in plane strain. ${ }^{7}$ The tri-crystal had near columnar grains with [001] orientations near the surface normal. The (compression) deformation of the $\sim 10 \times 15 \times 20 \mathrm{~mm}^{3}$ sample was carried out at $200^{\circ} \mathrm{C}$ in a channel die with the columnar and triplejunction directions along the zero strain direction of the channel..

\section{3-D TRIANGULATION MEASUREMENT TECHNIQUE}

For thin film samples, the experimental techniques discussed above provide micron resolution measurements simply by the use of a high-resolution translation stage to scan the twodimensional sample under the submicron beam. Three-dimensional samples, on the other hand, represent a much greater challenge because they require techniques to obtain spatial resolution along the direction of the beam. ${ }^{8}$ Figure 2 illustrates schematically the use of geometrical triangulation to achieve spatial resolution along the $\mathrm{x}$-ray beam. By collecting measurements at 
detector position 1 and position 2 without moving the sample, it is possible to determine the origin (along the beam direction) of diffraction peaks or features. With the use of a detector translation stage that is precise to $\sim 1$ micron, it is possible to achieve sub-pixel precision depthtriangulation. Through the use of $\sim 10$ different detector positions, it has been possible in some cases to achieve $\sim 0.1$ pixel resolution as discussed later. The centroid of a CCD image feature or group of features could be used in the triangulation as well as particular peak positions for the determination of the centroids of the local crystal structure producing the diffraction feature.
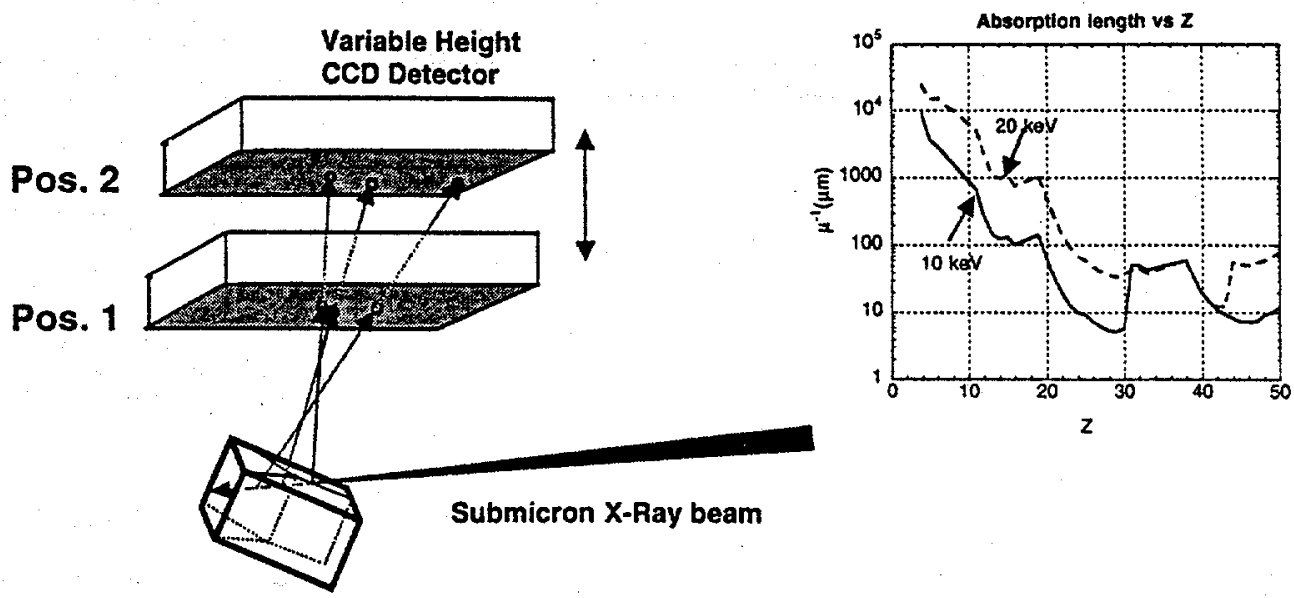

Fig. 2. Illustration of triangulation method for obtaining position resolution along the incident beam and a plot of the x-ray absorption length for elements at 10 and $20 \mathrm{keV}$.

\section{MICROBEAM DIFFRACTION MEASUREMENTS}

Figures 1,3 illustrate schematically the overall microbeam configurations used to perform diffraction measurements on the deformed sample. Figure 3 contains an optical photograph of the sample before and after deformation. ${ }^{7}$ The schematic drawing of the tri-crystal in the lower left corner indicates the orientation of the columnar-like grain structure and the triple-crystal junction in the measurements presented. The optical image is a photograph from a long
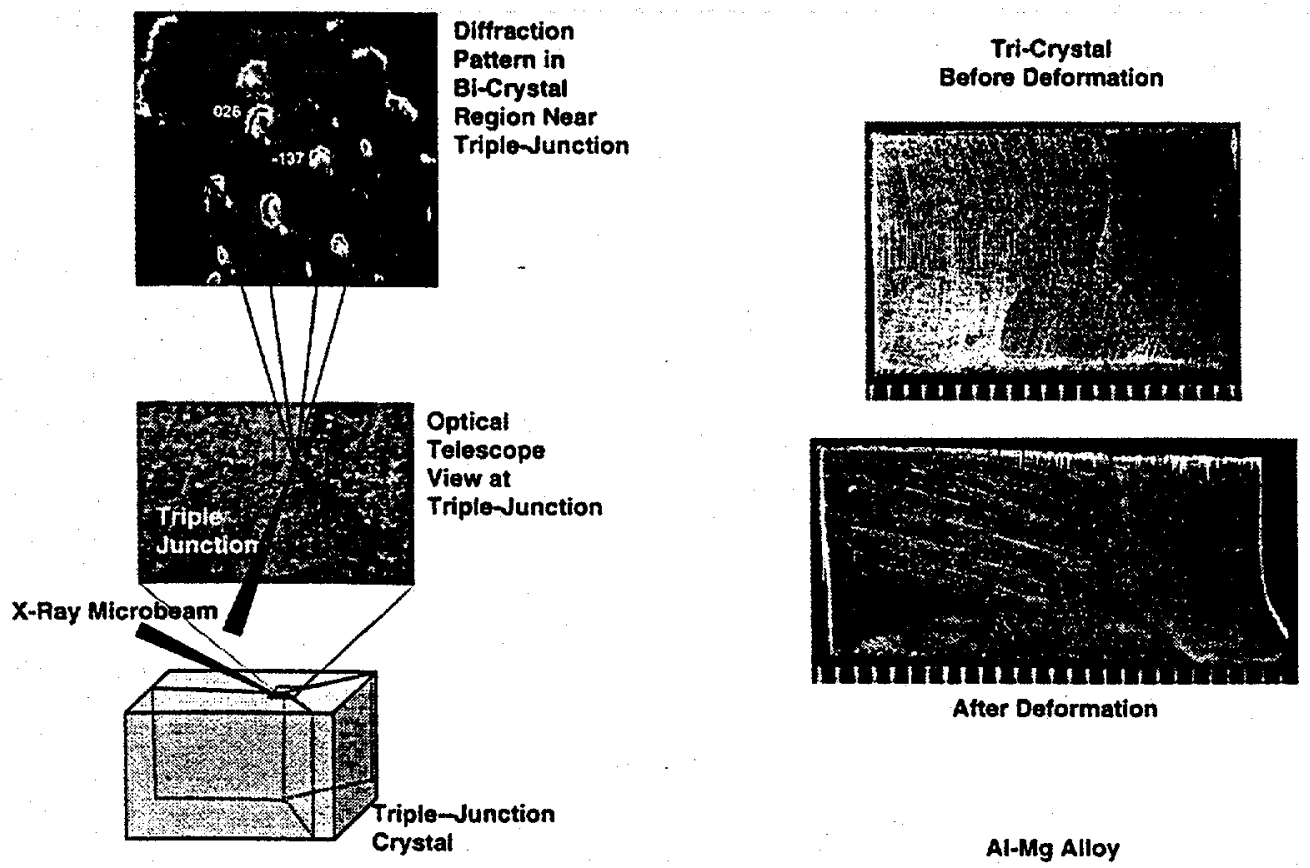

Fig. 3. Schematic view of microbeam diffraction from a deformed tri-crystal. The optical pictures at the right are before and after $20 \%$ plane strain; the pictures at the left depict the beam hitting the grain boundary producing a $\mathrm{CCD}$ diffraction pattern from both grains. 
focal-length telescope focused on the area immediately surrounding the position where the $\mathrm{x}$-ray microbeam strikes the sample. This telescope image contains an $\sim 150 \mu \mathrm{m}$ wide view of the sample area during the $\mathrm{x}$-ray measurement, which is used as a rough indicator of the position of the micro-beam with respect to optically visible features on the sample; precise positioning is inferred from the diffraction features.

The CCD image at the top of Fig. 3 contains a diffraction pattern collected with the $\mathrm{x}$-ray beam traversing the bi-crystal grain boundary to the left of the triple junction point, as indicated schematically in the lower left part of the figure. Diffraction spots from both grains in the bicrystal region are present in the image. At this beam position, the upper grain produces the arcshaped patterns and the lower grain gives rise to the shorter, vertically elongated spot pattern. For the measurements reported here, the sample normal was oriented at an angle of $45^{\circ}$ toward the beam; therefore, each diffraction pattern provides information on the deformation microstructure and sub-grain orientations in the deformed sample along a $0.7 \times 0.7 \mu \mathrm{m}^{2}$ crosssection line penetrating $0.25-0.5 \mathrm{~mm}$ into the sample at a $45^{\circ}$ angle.

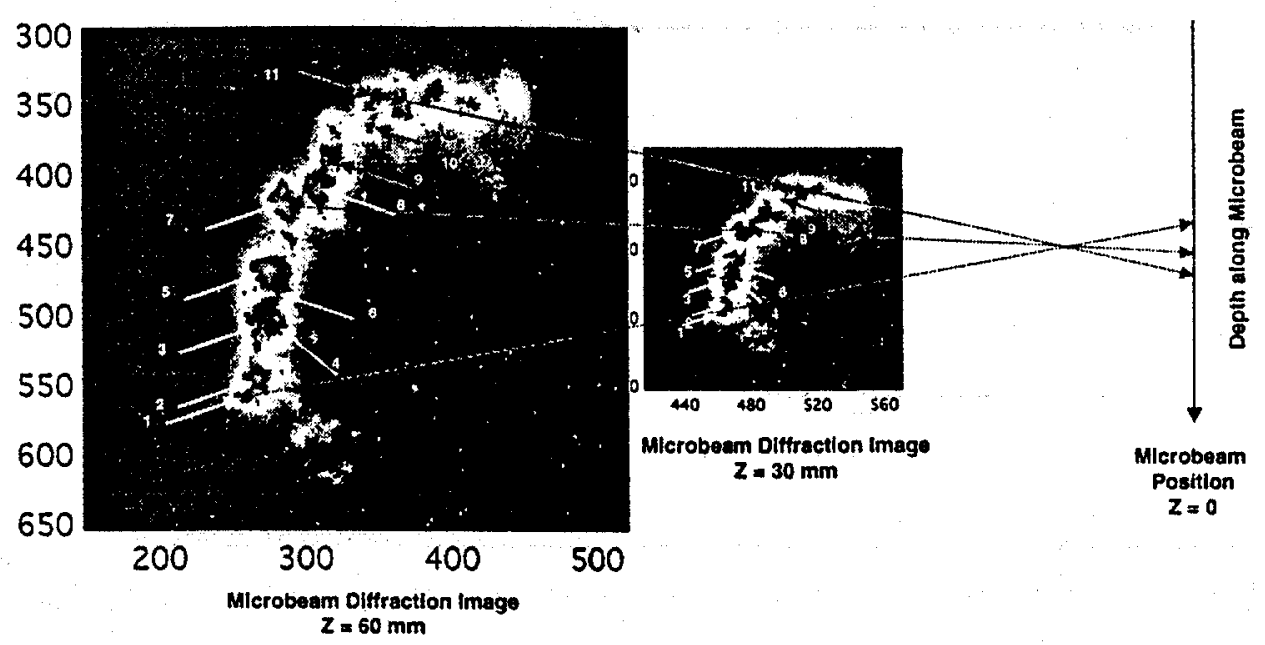

Fig. 4. CCD image of the (026) reflection in Fig. 3 for 30 and $60 \mathrm{~mm}$ from the microbeam height. The two images show schematically the method of triangulation to obtain the source point of the scattering for the diffraction features.

For the kinematical scattering conditions of a deformed sample, the diffraction patterns in the CCD image can be assumed to represent superpositions of the scattering from each crystallite illuminated along the $\mathrm{x}$-ray microbeam. Therefore, determination of the source point for each feature in the diffraction pattern yields a detailed characterization of the local crystal orientations and positions along the beam. Of course, features in particular (hkl) Bragg reflection have counterparts in each of the other Bragg reflections, thus providing a rich source of microstructural information. It should be recognized, though, that even micron size movements of the incident beam within a crystal lead to diffraction changes for samples with micron size substructure.

The 3-D triangulation measurements are performed by positioning the detector at varying distances above the micro-beam and collecting diffraction pattern images without sample motion. Figure 4 shows an expanded view of the (026) diffraction spot in Fig. 3 for detector distances of 60 and $30 \mathrm{~mm}$ from the beam. The deformation microstructure produces a 
diffraction pattern composed of $\sim 15$ major cluster-like structures distributed in the form of an arc. Simultaneous analysis of the diffraction features for each of the diffraction spots provides detailed, spatially resolved microstructural information along the $x$-ray microbeam. Techniques for such a comprehensive analysis are under development, but in this paper we restrict consideration to determination of the position (along the beam) of the origin of the diffraction features to demonstrate the triangulation technique.

The diffraction peak positions near the arrow heads in Fig. 4 were determined by fitting twodimensional Lorentzians to the corresponding peaks in diffraction images for 10 detector heights. These peak positions (in pixels) are plotted in Fig. 5 for each of the arrowed positions in Fig. 4, and similarly for four peaks in the diffraction spots corresponding to the lower grain of the bicrystal. Figure 5 shows the locus of the peak positions as a function of detector distance projected onto the plane containing the $x$-ray microbeam (i.e. $Z_{\mathrm{Det}}=0$ ). The expanded view in the right hand panel indicates the position along the beam that is identified as the source position for each of the diffraction peaks. Noting that the beam direction is implied to be top to bottom of the figure, it can be inferred that the beam traverses $\sim 50 \mu \mathrm{m}$ through the lower grain in Fig. 3 and $\sim 250 \mu \mathrm{m}$ into the upper grain up to the $11^{\text {th }}$ arrow in Fig. 4. It is important to note that the $\mathrm{x}$ position represents the microbeam position identically, so it is significant that the inferred $x$ positions from the triangulation are very close to that of the beam. In fact, since the $x$-position is determined in the same manner as the $y$-position, the deviations of the $x$-position origin from the exact beam position line provide a measure of the uncertainties in the determination of the $y$ position along the beam.

Using the deviations of the x-position and the fact that the two grains neither overlap nor leave a gap, we estimate the uncertainty along the beam to be less than 5 microns, and in the 2-3 micron range in many cases. Triangulation on weak peaks or peaks that become multiple peaks for

\section{Microbeam Diffraction Depth Triangulation}

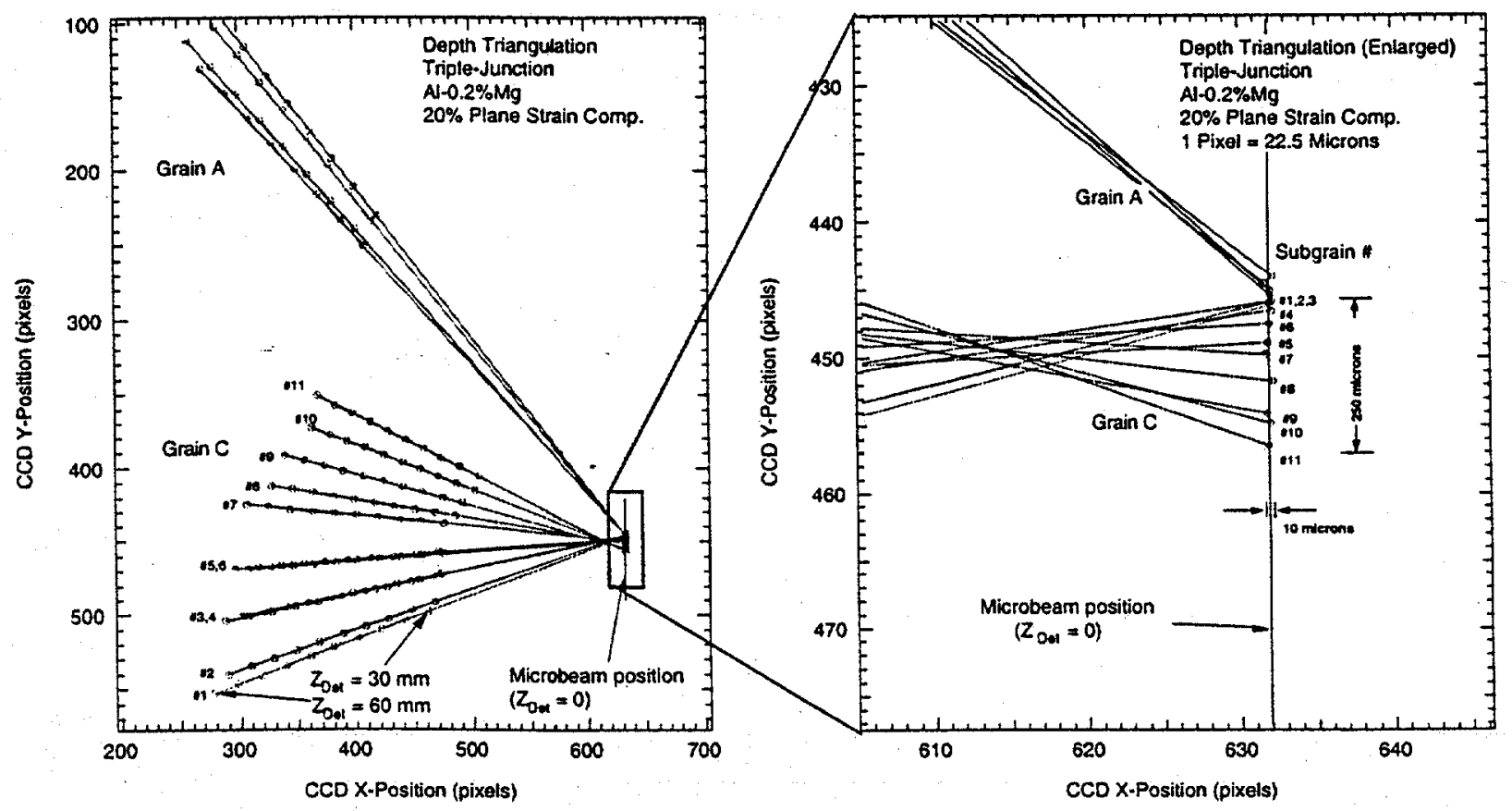

Fig. 5. Projection of diffraction peak positions for detector distances varying from $30-$ $60 \mathrm{~mm}$ onto the beam plane. Extrapolation of the lines to $Z=0$ determines the positions of the diffracting grains along the beam with an enlargement at right. 
larger detector distances do not achieve such high precision, of course, and will require fitting multiple peaks or additional triangulation techniques. However, the data presented indicate the triangulation precision for representative data.

The series of discrete clusters of diffraction intensity in Figs. 3,4 is consistent with the anticipated presence of layers of semi-aligned $\mathrm{Al}$ crystallites separated by dislocation walls with small angular rotations between layers. ${ }^{1,2}$ By identifying the corresponding peaks in a number of the (hkl) reflections, the orientation matrices ${ }^{3,4}$ of each of the individual diffraction features in Fig. 4 can be determined. As a result, it is possible to determine the angular separation and the axis of rotation between individual $\mathrm{Al}$ grains in their respective positions along the $\mathrm{x}$-ray beam. For instance, we have found that peaks at arrow positions 1 and 10 have relative rotations of 4.38 degrees around the $\sim[-2,3,3]$ direction. Both the direction and magnitude of the angular rotations for sample positions far removed from the grain boundary (say $\sim 1 \mathrm{~mm}$ ) were observed to be significantly different from those in Fig. 3, indicating the impact of the grain boundary on slip in the two grains.

\section{CONCLUSIONS}

A fundamental understanding of the mechanisms involved in the production of the well-known two-dimensional dislocation wall microstructure is of high interest both technologically and fundamentally. Continued development of the $\mathrm{x}$-ray microbeam techniques discussed here, including local stress/strain measurements, will provide new information regarding underlying issues involved in microstructural evolution. Microbeam $\mathrm{x}$-ray techniques provide mesoscale information complementary to that presently available by TEM and electron backscattering OIM techniques. In particular, microbeam $\mathrm{x}$-ray diffraction provides non-destructive bulk measurements and they provide the possibility of measuring local stress distributions under loaded and/or dynamic conditions.

\section{ACKNOWLEDGMENTS}

Research sponsored by the U.S. Department of Energy under contract DE-AC05-96OR22464 with Lockheed Martin Energy Research Corp. The x-ray measurements were performed on the MHATT-CAT beam line at the APS. The APS is supported by the DOE Office of Energy Research under contract W-31-109-ENG-38.

\section{REFERENCES}

1. P. Hähner and M. Zaiser, Acta Mater. 45, 1067 (1997).

2. N. Hansen and D. A. Hughes, Phys. Stat. Sol. (b) 149, 155 (1995).

3. J.-S. Chung and G.E. Ice, J. Appl. Phys., 86, 5249 (1999).

4. J.-S. Chung, N. Tamura, G. E. Ice, B. C. Larson, J. D. Budai, and W. Lowe, Mat. Res. Soc. Proc. 563, 169 (1999).

5. N. Tamura, J.-S. Chung, G. E. Ice, B. C. Larson, J. D. Budai, J. Z. Tischler, M. Yoon, E. L. Williams, and W. Lowe, Mat. Res. Soc. Proc. 563, 175 (1999).

6. G. E. Ice, J.-S. Chung, W. Lowe, E. L. Williams, and J. Edelman, Rev. Sci. Inst. (submitted).

7. H. Weiland and R. Becker, in Proceeding 20th Riso Intern. Symp. on Mat. Sci.: Deformation-induced Microstructures: Analysis and Relation to Properties, edited by $\mathrm{T}$. Leffer and O.P. Pederson, Riso Intern. Laboratory, Roskilde, Denmark 1999, p. 213.

8. D.P. Piotrowski, S.R. Stock, A. Guvenilir, J.D. Haase, and Z.U. Rek, Mat. Res. Soc. Symp. 437, 125 (1996). 